\title{
THE WEDDERBURN PRINCIPAL THEOREM IN BANACH ALGEBRAS
}

\section{CHESTER FELDMAN}

The Principal Theorem of Wedderburn for a finite-dimensional algebra $A$ states that $A$ is the vector space direct sum of its radical $R$ and an algebra isomorphic to $A / R$. It will be shown that the corresponding theorem is not true for all Banach algebras, but that it is true with certain restrictions.

The terminology of Jacobson [3] ${ }^{1}$ will be followed for radical, quasi-inverse, and quasi-regular. The notations $x \bigcirc y=x+y+x y$ and $x^{\prime}$ for the quasi-inverse of $x$ will also be employed.

Definition 1. A Banach algebra is a complete normed linear space which is also an algebra over the complex numbers satisfying $\|x y\|$ $\leqq\|x\|\|y\|$.

All the following results are proved for real algebras in [1] by the same methods.

To show that the Wedderburn theorem does not hold for an arbitrary Banach algebra, consider the commutative algebra $A$ which is the completion of the algebra of all finite sums

$$
\sum_{i=1}^{n} \alpha_{i} e_{i}+\beta r
$$

where $\alpha_{i}$ and $\beta$ are complex, $e_{i}$ are mutually orthogonal idempotents, $r^{2}=0, e_{i} r=r e_{i}=0$, and

$$
\left\|\sum \alpha_{i} e_{i}+\beta r\right\|=\max \left\{\left[\sum\left|\alpha_{i}\right|^{2}\right]^{1 / 2},\left|\beta-\sum \alpha_{i}\right|\right\} .
$$

It is easy to show this defines a norm, but it is also necessary to verify that $\|x y\| \leqq\|x\|\|y\|$. Let $x=\sum \alpha_{i} e_{i}+\gamma r, y=\sum \beta_{i} e_{i}+\nu r$. Then $x y=\sum \alpha_{i} \beta_{i} e_{i} ;\|x y\|=\max \left\{\left[\sum\left|\alpha_{i} \beta_{i}\right|^{2}\right]^{1 / 2},\left[\sum \alpha_{i} \beta_{i} \mid\right\}\right.$. By the Cauchy inequality,

$$
\left|\sum \alpha_{i} \beta_{i}\right| \leqq \sum\left|\alpha_{i} \beta_{i}\right| \leqq\left[\sum\left|\alpha_{i}\right|^{2}\right]^{1 / 2}\left[\sum\left|\beta_{i}\right|^{2}\right]^{1 / 2} .
$$

Together with $\sum\left|\alpha_{i} \beta_{i}\right|^{2} \leqq \sum\left|\alpha_{i}\right|^{2} \sum\left|\beta_{i}\right|^{2}$ this shows $\|x y\| \leqq\|x\|\|y\|$. Hence $A$ is a Banach algebra.

$A / R$ is the algebra of all sequences $\sum \alpha_{i} u_{i}$ where $u_{i}^{2}=u_{i}, \alpha_{i}$ are complex, and $\left\|\sum \alpha_{i} u_{i}\right\|=\left[\sum\left|\alpha_{i}\right|^{2}\right]^{1 / 2}<\infty . A / R$ contains the element $x=\sum_{i=1}^{\infty} i^{-1} u_{i}$ since $\sum i^{-2}=\pi^{2} / 6$, but there is no element

Received by the editors November 27, 1950.

1 Numbers in brackets refer to the references cited at the end of the paper. 
$\sum i^{-1} e_{i}$ in $A$, for $\sum i^{-1}$ diverges. Therefore there is no subalgebra of $A$ isomorphic to $A / R$.

It can be shown [1] that the radical of $A$ is one-dimensional. Thus no restriction on the dimension of the radical will suffice. However it will now be shown that it is sufficient for $A / R$ to be finite-dimensional.

Theorem 1. If $A$ is a Banach algebra, $R$ its radical, and $A / R$ is finite-dimensional, then there is a subalgebra $S$ of $A$ isomorphic and homeomorphic to $A / R$. $A$ is the vector space direct sum $S+R$.

Lemma 1. If $A$ is a Banach algebra, $R$ its radical, and $\left\{u_{i}\right\} a$ denumerable set of pairwise orthogonal idempotents of $A / R$, then there exist idempotents $e_{i}$ in $A$ mapping on $u_{i}$ via $A \rightarrow A / R$, and the $e_{i}$ are pairwise orthogonal.

The proof is by induction. Let $a_{1}$ be an element of $A$ mapping on the class $u_{1}$. Then $a_{1}^{2}-a_{1}=r_{1}$ in $R$ by hypothesis. For any $r$ in $R$ there exists $(1+4 r)^{-1 / 2}=1-2 r+6 r^{2}-20 r^{3}+\cdots$ since $\left\|r^{n}\right\|^{1 / n} \rightarrow 0$ [2] guarantees convergence of this series. Define $e_{1}=\left(2 a_{1}-1\right)\left[2(1+4 r)^{1 / 2}\right]^{-1}$ $+1 / 2=a_{1}\left(1-2 r+6 r^{2}-\cdots\right)+\left(r-3 r^{2}+10 r^{3}-\cdots\right)$. Then $e_{1}^{2}=e_{1}$ and $e_{1}$ maps on $u_{1}$ since $a_{1}$ does. Assume there exist $e_{1}, \cdots, e_{t-1}$ such that $e_{i}^{2}=e_{i}, e_{i} e_{j}=0=e_{j} e_{i}$ for $i \neq j$, and $e_{i} \rightarrow u_{i}, i=1,2, \cdots, t-1$. Define $f=\sum_{i=1}^{t-1} e_{i}$. Then $f^{2}=f, e_{i} f=f e_{i}$. Let $b_{t}$ be any element such that $b_{t} \rightarrow u_{t}$. Define $a_{t}=(1-f) b_{t}(1-f)$. Then $e_{i} a_{t}=a_{t} e_{i}=0$, and $a_{t} \rightarrow u_{t}$ since $f b_{t} \rightarrow 0, b_{t} f \rightarrow 0$, and $f b_{t} f \rightarrow 0$. Hence $a_{t}^{2}-a_{t}=r_{t}$ in $R$ and $e_{i} r_{t}=r_{t} e_{i}=0$, $i=1,2, \cdots, t-1$. Define $e_{t}=\left(2 a_{t}-1\right)\left[2\left(1+4 r_{t}\right)^{1 / 2}\right]^{-1}+1 / 2$. Then $e_{t}^{2}=e_{t}, e_{t} \rightarrow u_{t}$, and $e_{t} e_{i}=e_{i} e_{t}=0$ since $e_{i} a_{t}=e_{i} r_{t}=0$. This completes Lemma 1.

Lemma 2. If $A / R$ contains a ring direct sum $M_{1} \oplus M_{2} \oplus \cdots \oplus M_{t}$ of total matric algebras $M_{i}$, then $A$ contains a ring direct sum of total matric algebras $S_{i} \rightarrow M_{i}$ via $A \rightarrow A / R$.

Consider first a single matric algebra $M \subset A / R$, where $M$ is generated over the complexes by $u_{i j}, u_{i i}$ are pairwise orthogonal idempotents, $u_{i j} u_{j k}=u_{i k}$, and $u_{i j} u_{k s}=0$ for $k \neq j$. Since there are a finite number of $u_{i i}$, by Lemma $1 A$ contains idempotents $e_{i i} \rightarrow u_{i i}$ with $e_{i i} e_{j j}=e_{j j} e_{i i}=0$ for $i \neq j$. Choose an element $v_{i 1} \rightarrow u_{i 1}$ and an element $v_{1 j} \rightarrow u_{1 j}$. Since $u_{i i} u_{i 1} u_{11}=u_{i 1}$ and $u_{11} u_{1 j} u_{j j}=u_{1 j}$, $v_{i 1}$ may be chosen in $e_{i i} A e_{11} ; v_{1 j}$ may be chosen in $e_{11} A e_{j j}$. Then $v_{1 j} v_{j 1} \rightarrow u_{1 j} u_{j 1}=u_{11}$. Hence $v_{1 j} v_{j 1}=e_{11}+a_{j}$ where $a_{j}$ is in $R \cap e_{11} A e_{11}$. By [3], $a_{j}^{\prime}$ exists. $\left(e_{11}+a_{j}^{\prime}\right)\left(e_{11}+a_{j}\right)$ $=e_{11}+a_{j}^{\prime} e_{11}+e_{11} a_{j}+a_{j}^{\prime} a_{j}=e_{11}$ since $a_{j}^{\prime}=\sum\left(-a_{j}\right)^{n}$ is also in $e_{11} A e_{11}$. Define $e_{i j}=e_{i 1} e_{1 j}$. Then $e_{i j} e_{j k}=e_{i k}$ and $e_{i j} e_{k k}=0$ for $j \neq k h$. Clearly $e_{i j}$ is 
in $A$ and $e_{i j} \rightarrow u_{i j}$. Thus $A$ contains a total matric algebra $\left(e_{i j}\right)$ isomorphic to $M$. The sum of the algebras $S_{i}$ so constructed for each $M_{i}$ is the ring direct sum since the basis elements are constructed from mutually orthogonal idempotents. This completes Lemma 2.

Proof of THEOREM. $A / R$ is the direct sum of a finite number of finite-dimensional total matric algebras over the complex numbers. Hence $A$ contains a subalgebra $S \cong A / R$. Since the isomorphism $S \rightarrow A / R$ is continuous, it is a homeomorphism. $S$ is semi-simple; so $S \cap R=0$. Therefore $S+R$ is a vector space direct sum.

When $A / R$ is not finite-dimensional the theorem can still be proved if $R$ is finite-dimensional and $A / R$ is a well known type of algebra most generally defined in [4] as follows:

Definition 2. The $B(\infty)$ direct sum of a denumerable number of algebras $A_{i}$ is the completion in a specified norm of the algebra of all sequences $\left\{a_{i}\right\}$ such that $a_{i}$ in $A_{i}$ are 0 for all but a finite number of $i$.

THEOREM 2. If $A$ is a Banach algebra, the radical $R$ of $A$ is finitedimensional, and $A / R$ is the $B(\infty)$ direct sum of finite-dimensional total matric algebras, then $A$ is a vector space direct sum, $A=B+C+D$, where $B$ is finite-dimensional, $B C=C B=0$, every idempotent of $C$ mapping on an element in the basis of $A / R$ is orthogonal to $R$, and $D \subset R$. When $A$ is commutative, $D=0$ and $A$ is a ring direct sum of $B$ and $C$.

Let $n$ be the dimension of $R$. Then there are at most $n$ distinct primitive orthogonal idempotents $e_{k}$ and $n$ distinct primitive orthogonal idempotents $e_{s}$ of $A$ for which $e_{k} r_{k} \neq 0$ and $r_{s} e_{s} \neq 0$ for any $r_{k}$ and $r_{\text {, in }} R$. Otherwise

$$
e_{n+1} r_{n+1}=\sum_{k=1}^{n} \alpha_{k} e_{k} r_{k}, \quad r_{n+1} e_{n+1}=\sum_{s=1}^{n} \beta_{8} r_{8} e_{s}
$$

for complex $\alpha_{k}$ and $\beta_{s}$, since any $n+1$ elements of $R$ are linearly dependent. However,

$$
\begin{aligned}
& e_{n+1}\left(e_{n+1} r_{n+1}\right)=e_{n+1} r_{n+1}=\sum \alpha_{k} e_{n+1} e_{k} r_{k}=0, \\
& \left(r_{n+1} e_{n+1}\right) e_{n+1}=r_{n+1} e_{n+1}=\sum \beta_{s} r_{s} e_{s} e_{n+1}=0 .
\end{aligned}
$$

Hence there are at most $2 n$ primitive orthogonal idempotents $e_{j}$ for which $e_{j} R \neq 0$ or $R e_{j} \neq 0$.

Let $\left\{u_{i j}\right\}$ be a basis for the matric algebras of $A / R$. Choose a fixed set of $e_{i j}$ constructed as in Lemma 2 to map on $u_{i j}$, and number the set so that $e_{j}=e_{j j}, j=1, \cdots, s$, are all idempotents of the set $\left\{e_{i j}\right\}$ which are not orthogonal to the radical. Define $e=\sum_{j=1}^{s} e_{j}, B=e A e$, $C=(1-e) A(1-e)$, and $D=e A(1-e)+(1-e) A e$. Then $A=B+C+D$ 
is the usual two-sided Peirce decomposition of $A$. Obviously $B C$ $=C B=0$.

If $A$ is commutative, $e(1-e)=0$; so $D=0$. Therefore $A$ is a ring direct sum, $A=B \oplus C$.

Note that if $e_{i}=e_{i i}$ is an idempotent of $\left\{e_{i j}\right\}$ which is orthogonal to $R$ and $e_{k}=e_{k k}$ is an idempotent of $\left\{e_{i j}\right\}$ which maps on $u_{k}=u_{k k}$ in the same matric algebra as $u_{i i}$, then $e_{k k}$ is also orthogonal to $R$, since by Lemma 2 there exist $e_{i k}$ and $e_{k i}$ such that $e_{k i} e_{i i} e_{i k}=e_{k k}$. Then $e_{k k} R$ $=e_{k i} e_{i i} e_{i k} R=0$, and $R e_{k k}=R e_{k i} e_{i i} e_{i k}=0$.

Let $u$ be the image of $e$ under $A \rightarrow A / R$. Then $u$ is the sum $u=I_{1}$ $+\cdots+I_{n}$ where $I_{m}$ is the unit element of a matric algebra in $A / R$. Now $D \rightarrow u(A / R)(1-u)+(1-u)(A / R) u$. Since $u$ commutes with $A / R, D \rightarrow 0$. Therefore $D \subset R . e A e / R$ is finite-dimensional and $R$ is finite-dimensional. Therefore $e A e$ is finite-dimensional. All idempotents of $\left\{e_{i j}\right\}$ not orthogonal to $R$ are in $B$; so all idempotents of $\left\{e_{i j}\right\}$ in $C$ are orthogonal to $R$. This completes Theorem 2 .

The Principal Theorem of Wedderburn is known for finite-dimensional algebras, so $B=S_{1}+R_{1}$. If it can be proved that $C=S_{2}+R_{2}$, then it is proved for $A$; for $S=S_{1}+S_{2}$ is a subalgebra, and it follows from $B C=C B=0$ that $S_{1} S_{2}=S_{2} S_{1}=0$, which implies $S_{1}+S_{2} \cong A / R$.

A $C^{*}$-algebra is a Banach algebra with a conjugate linear involution $x \rightarrow x^{*}$ such that $\left(x x^{*}\right)^{\prime}$ exists for all $x$ and $\left\|x x^{*}\right\|=\|x\|^{2}$. It is proved in [4] that a completely continuous $C^{*}$-algebra is the $B(\infty)$ direct sum of finite-dimensional total matric algebras.

Theorem 3. If $A / R$ is a completely continuous $C^{*}$-algebra and $R$ is finite-dimensional, then $A$ is a vector space direct sum, $A=S+R$, of $R$ and an algebra $S$ isomorphic and homeomorphic to $A / R$.

Theorem 2 applies to give $A=B+C+D$. The remark above implies a continuous isomorphism between $S_{1}$ and $B / R_{1}$. By the closed graph theorem this is a homeomorphism; so it remains to prove the theorem only for the algebra $C$ in which every idempotent of the set $\left\{e_{i j}\right\}$ is orthogonal to $R$. It will thus be assumed that all idempotents in the set $\left\{e_{i j}\right\}$ are orthogonal to $R$.

LEMma 3. All elements of $\left\{e_{i j}\right\}$ are orthogonal to $R$.

Since $e_{i j}=e_{i i} e_{i j}=e_{i j} e_{j j}$, and it has been assumed that all idempotents are orthogonal to $R$, it is clear that all $e_{i j}$ are.

Lemma 4. $\left\|e_{i j}\right\|=\left\|u_{i j}\right\|=1$.

By [5, Theorem 10] and [4] the basis $\left\{u_{i j}\right\}$ may be chosen so that $u_{i j}^{*}=u_{j i}$. 


$$
\begin{aligned}
& \left\|u_{i i} u_{i j}^{*}\right\|=\left\|u_{i i}^{2}\right\|=\left\|u_{i i}\right\|=\left\|u_{i i}\right\|^{2} \text {. Hence }\left\|u_{i i}\right\|=1 \text {. } \\
& \left\|u_{i j} u_{i j}^{*}\right\|=\left\|u_{i j}\right\|^{2}=\left\|u_{i j} u_{j i}\right\|=\left\|u_{i i}\right\|=1 \text {. Hence }\left\|u_{i j}\right\|=1 \text {. By definition, } \\
& \inf _{r \in R}\left\|e_{i i}+r\right\|=\left\|u_{i i}\right\|=1 \text {. }
\end{aligned}
$$

Let $n$ be the dimension of $R$. Then $r^{n+1}=0$.

$$
\begin{aligned}
\left\|\left(e_{i i}+r\right)^{n+1}\right\| & =\left\|e_{i i}^{n+1}+(n+1) e_{i i}^{n}+\cdots+r^{n+1}\right\| \\
& =\left\|e_{i i}\right\| \leqq\left\|e_{i i}+r\right\|^{n+1} .
\end{aligned}
$$

For any $\epsilon>0$ there is an $r$ in $R$ for which $\left\|e_{i i}+r\right\|^{n}<1+\epsilon$. Hence $\left\|e_{i i}\right\| \leqq 1$. Since $\left\|e_{i i}\right\| \leqq\left\|e_{i i}\right\|^{2},\left\|e_{i i}\right\|=1$. Now

$$
\begin{gathered}
\inf _{r \in R}\left\|e_{i 1}+r\right\|=\left\|u_{i 1}\right\|=1, \\
\inf _{r \in R}\left\|e_{i j}+r\right\|=\left\|u_{i j}\right\|=1, \\
e_{i 1}=\left(e_{i 1}+r\right) e_{11}, \\
\left\|e_{i 1}\right\| \leqq\left\|e_{i 1}+r\right\|\left\|e_{11}\right\|=\left\|e_{i 1}+r\right\|, \\
e_{1 j}=e_{11}\left(e_{1 j}+r\right), \\
\left\|e_{1 j}\right\| \leqq\left\|e_{11}\right\|\left\|e_{1 j}+r\right\|=\left\|e_{1 j}+r\right\| .
\end{gathered}
$$

This shows $\left\|e_{i 1}\right\| \leqq 1$ and $\left\|e_{1 j}\right\| \leqq 1$. The mapping $e_{i j} \rightarrow u_{i j}$ depresses the norm.

$$
\left\|e_{i j}\right\| \leqq\left\|e_{i 1}\right\|\left\|e_{1 j}\right\| \leqq 1
$$

Therefore $\left\|e_{i j}\right\|=1$.

Proof of theorem. $A / R$ is the $B(\infty)$ direct sum of finite-dimensional total matric algebras $M_{i}$. By Lemma $4, A$ contains a subalgebra $S_{i}$ equivalent to $M_{i}$. It will be shown that the map of any finite sum $\sum_{i=1}^{t} N_{i}, N_{i}$ in $S_{i}$, into $A / R$ is an isometry. Suppose $N_{i} \rightarrow \bar{N}_{i}$ in $M_{i}$, and $I_{i}$ is the identity matrix of $S_{i}$. Since $A / R$ is a $C^{*}$-algebra, $\left(\bar{I}_{1} \bar{I}_{1}^{*}\right)^{*}=\bar{I}_{1}=\bar{I}_{1} \bar{I}_{1}^{*},\left\|\bar{I}_{1}\right\|=\left\|\bar{I}_{1} \bar{I}_{1}^{*}\right\|=\left\|\bar{I}_{1}\right\|^{2}$; so $\left\|\bar{I}_{1}\right\|=1$. Furthermore

$$
\begin{aligned}
& \left\|\bar{I}_{1}+\cdots+\bar{I}_{t}\right\|=\left\|\left[\left(\bar{I}_{1}+\cdots+\bar{I}_{t}\right)\left(\bar{I}_{1}^{*}+\cdots+\bar{I}_{t}^{*}\right)\right]^{*}\right\|, \\
& \left\|\bar{I}_{1}+\cdots+\bar{I}_{t}\right\|=\left\|\bar{I}_{1}+\cdots+\bar{I}_{t}\right\|^{2}=1 .
\end{aligned}
$$

Define $I=I_{1}+\cdots+I_{t}$. Then

$$
\begin{aligned}
& \inf _{r \in R}\|I+r\|=\|\bar{I}\|=1 \\
& \left\|(I+r)^{n+1}\right\|=\|I\| \leqq\|I+r\|^{n+1} .
\end{aligned}
$$

Hence $\|I\|=1$, and similarly 


$$
\begin{aligned}
\inf _{r \in R}\left\|\sum_{i=1}^{t} N_{i}+r\right\| & =\left\|\sum \bar{N}_{i}\right\|, \\
I\left(\sum N_{i}+r\right) & =\sum N_{i}, \\
\left\|\sum N_{i}\right\| \leqq\|I\|\left\|\sum N_{i}+r\right\| & =\left\|\sum N_{i}+r\right\|, \\
\left\|\sum N_{i}\right\| & \leqq\left\|\sum \bar{N}\right\| .
\end{aligned}
$$

Since the mapping $A \rightarrow A / R$ depresses norms,

$$
\left\|\sum N_{i}\right\|=\left\|\sum \bar{N}_{i}\right\| \text {. }
$$

This shows that the mapping of any finite sum $\sum_{i=1}^{t} N_{i}$ into $A / R$ is an isometry. Let $S$ be the $B(\infty)$ direct sum of the subalgebras $S_{i}$ of $A$. Since $A$ is complete, $S \subset A$. A dense subset of $S$ maps isometrically and isomorphically onto a dense subset of $A / R$; therefore $S$ is isomorphic and isometric to $A / R$. This proves Theorem 3 .

The theorem will now be proved for an algebra in which the mapping $A \rightarrow A / R$ depresses the norm as little as possible.

Definition 3. An $l_{1}$ algebra is the commutative Banach algebra of all sums $\sum \alpha_{i} u_{i}$, where $\alpha_{i}$ are complex, $u_{i}$ are a denumerable number of primitive orthogonal idempotents, and $\left\|\sum \alpha_{i} u_{i}\right\|=\sum_{\mid}^{\prime} \alpha_{i} \mid<\infty$.

Theorem 4. If $A / R$ is an $l_{1}$ algebra and $R$ is finite-dimensional, then $A=S+R$ where $S$ is a subalgebra of $A$ isomorphic and homeomorphic to $A / R$.

As in Theorem 3 it is sufficient to consider an algebra $A$ in which each idempotent $e_{i}$ is orthogonal to $R$.

There exist pairwise orthogonal idempotents $e_{i} \rightarrow u_{i}$ by Lemma 1 . The proof of Lemma 4 shows $\left\|e_{i}\right\|=1$. For any $x=\sum \alpha_{i} e_{i}$ in $A$, $\|x\| \leqq\left\|\sum \alpha_{i} e_{i}\right\| \leqq \sum\left|\alpha_{i}\right|\left\|e_{i}\right\|=\sum\left|\alpha_{i}\right|=\left\|\sum \alpha_{i} u_{i}\right\|$. Now $x \rightarrow \sum \alpha_{i} u_{i}$, and the mapping $A \rightarrow A / R$ decreases norms. Hence $\|x\|=\sum\left|\alpha_{i}\right|$, that is, the mapping is an isometry on the completion $S$ of the subalgebra of $A$ generated by the $e_{i}$. Therefore $S$ is isometric and isomorphic to $A / R$ and $A=S+R$. This completes the proof.

In all the previous theorems the completion of the algebra generated by elements mapping on basis elements of $A / R$ is disjoint from the radical. The following theorem shows this property is the essential one.

Theorem 5. Suppose $A$ is a Banach algebra, that the radical $R$ is finite-dimensional, that $A / R$ is the $B(\infty)$ sum of finite-dimensional total matric algebras, that $S$ is the $B(\infty)$ sum in $A$ of the matric algebras isomorphic to those of $A / R$, and that $S \cap R=0$. Then $S$ is isomorphic and homeomorphic to $A / R$, and $A$ is the vector space direct sum $S+R$. 
$S$ is complete and $R$ is complete since the radical of a Banach algebra is closed. $R$ is finite-dimensional so $S+R$ is complete. Also $(S+R) / R$ is complete; hence $A \rightarrow A / R$ maps $S+R$ onto $A / R$. $S \cap R$ $=0$ implies $(S+R) / R=S$. Therefore $S \cong A / R$. The mapping $S \rightarrow A / R$ is $1-1$ and continuous. By the closed graph theorem, $S$ is homeomorphic to $A / R$. Suppose $a$ in $A$ maps on $[a]$ in $A / R$. Then there is an $s$ in $S$ which maps on $[a]$. Thus $a-s=r$ in $R$. Every $a=s+r$. Since $S$ is semi-simple, $A=S+R$.

\section{REFERENCES}

1. C. Feldman, Real Banach algebras, University of Chicago Dissertation, University of Chicago Library Microfilm T. 832.

2. I. Gelfand, Normierte Ringe, Rec. Math. (Mat. Sbornik) N.S. vol. 9 (1941) pp. 3-24.

3. N. Jacobson, The radical and semi-simplicity for arbitrary rings, Amer. J. Math. vol. 67 (1945) pp. 300-320.

4. I. Kaplansky, Normed algebras, Duke Math. J. vol. 16 (1949) pp. 399-418.

5. —, Dual rings, Ann. of Math. vol. 49 (1948) pp. 689-701.

SOUTH BEND, IND. 